\title{
Microstructural, magnetic anisotropy, and magnetic domain structure correlations in epitaxial FePd thin films with perpendicular magnetic anisotropy
}

\author{
J. R. Skuza ${ }^{1}$, C. Clavero ${ }^{2}$, K. Yang ${ }^{2}$, B. Wincheski ${ }^{3}$, and R. A. Lukaszew ${ }^{1,2}$ \\ ${ }^{1}$ Department of Physics, College of William \& Mary, Williamsburg, Virginia 23187 USA \\ ${ }^{2}$ Department of Applied Science, College of William \& Mary, Williamsburg, Virginia 23187 \\ USA \\ ${ }^{3}$ Nondestructive Evaluation Sciences Branch, NASA Langley Research Center, Hampton, VA \\ 23681 USA
}

Index Terms - Magnetic domains, perpendicular magnetic anisotropy, perpendicular magnetic recording, thin films

$L 1_{0}$-ordered FePd epitaxial thin films were prepared using dc magnetron sputter deposition on $\mathrm{MgO}$ (001) substrates. The films were grown with varying thickness and degree of chemical order to investigate the interplay between the microstructure, magnetic anisotropy, and magnetic domain structure. The experimentally measured domain size/period and magnetic anisotropy in this high perpendicular anisotropy system were found to be correlated following the analytical energy model proposed by Kooy and Enz that considers a delicate balance between the domain wall energy and the demagnetizing stray field energy. 


\section{Introduction}

Magnetic domain analysis is relevant to further understand the relationship between microstructure and magnetic anisotropy in magnetic materials, particularly when the materials are in thin film form. ${ }^{1}$ Of particular interest in the last decade are thin films that exhibit strong perpendicular magnetic anisotropy (PMA) because of their possible application in next generation magnetic recording, magneto-optic recording, and patterned media. ${ }^{2}$ These strong PMA thin films must have a uniaxial quality factor $\left(Q_{u}=K_{u} / K_{d}\right)$ greater than 1 , with first order uniaxial anisotropy constants $\left(K_{u}\right)$ in the range of $10^{6} \mathrm{~J} / \mathrm{m}^{3}$ and where the stray field energy coefficient $\left(K_{d}\right)$ is equal to $\mu_{0} M_{s}^{2} / 2\left(\mathrm{~J} / \mathrm{m}^{3}\right)$. The FePd binary alloys belong to this category because they exhibit the $L 1_{0}$ ordered phase ( $\mathrm{CuAu}$ I structure), which is responsible for strong PMA when the stoichiometry of the alloy is near $1: 1{ }^{3}$ The crystallographic structure of the $L 1_{0}$ ordered phase is characterized by alternating Fe and Pd atomic layers along a cubic stacking direction, which as a consequence suffers a tetragonal distortion. This tetragonal distortion induces a strong PMA in chemically ordered thin films when the layering (structural anisotropy) is parallel to the film plane. $K_{u}$ in these materials is an order of magnitude greater than Co-Cr based alloys presently used in recording media and is due to large spin-orbit coupling of the paramagnetic $\mathrm{Pd}$ atoms and a strong hybridization of their $4 d$ bands with the highly polarized Fe $3 d$ bands. $^{4}$

High squareness in the perpendicular $\mathrm{M}-\mathrm{H}$ loop and reduced magnetic domain size are the key properties for recording media with low noise. ${ }^{5}$ Therefore, understanding domain size correlations with magnetic anisotropy is an important step towards development of optimized high density perpendicular magnetic recording media. In the present study, we present the correlation between magnetic domain patterns and magnetic anisotropy in a system where the latter can be tailored via $L 1_{0}$ chemical ordering. Thus, we have prepared and characterized epitaxial FePd thin films directly on MgO substrates with various FePd thicknesses and degrees of $L 1_{0}$ chemical order to investigate the interplay between microstructure, magnetic anisotropy, and magnetic domain structure. We have found good agreement between the experimentally measured values of the magnetic anisotropy and magnetic domain size/period in accordance with the analytical energy model proposed by Kooy and Enz (K-E), ${ }^{6}$ where the magnetic anisotropy has been correlated with the actual microstructure of the samples.

\section{Experimental Conditions}

Epitaxial FePd films were grown directly on $\mathrm{MgO}(001)$ substrates in an ultra-high vacuum (UHV) deposition system with base pressure in the low $10^{-9}$ Torr range. The majority of previous work has focused on FePd growth onto $\mathrm{Cr} / \mathrm{Pd}$, Pd, or Pt buffer layers to improve the FePd $L 1_{0}$ crystal orientation. ${ }^{7,8,9,10}$ However, the high temperatures needed during growth or postdeposition annealing to form the $L 1_{0}$ phase cause atomic diffusion at the FePd/buffer interface. The FePd film growth presented here was carried out using dc magnetron sputtering by codeposition from high purity (99.99\%) Fe and Pd targets at an Ar pressure of 5 mTorr. The FePd deposition rate was $0.26 \AA / \mathrm{s}$ ( $4.2 \mathrm{ML} / \mathrm{min}$.). Two series of samples were grown on $\mathrm{MgO}(001)$ substrates: a series of FePd films with fixed thickness $(40 \mathrm{~nm})$ where the deposition temperature was varied from room temperature (RT) to $600{ }^{\circ} \mathrm{C}$ and a series where the thickness was varied from $5-80 \mathrm{~nm}$ and grown at the temperature at which higher chemical ordering was found (450 
$\left.{ }^{\circ} \mathrm{C}\right)$. $\mathrm{MgO}(001)$ substrates were ultrasonically cleaned in subsequent baths of acetone and methanol for 10 minutes each. Before growth, the substrates were UHV annealed at $700{ }^{\circ} \mathrm{C}$ for 30 minutes to degas and re-crystallize the surface layers of the substrate, thus improving the epitaxial growth of FePd on MgO(001). ${ }^{11}$ The FePd films were capped with a $5 \mathrm{~nm}$ Au layer deposited at RT and at an Ar pressure of 5 mTorr with a deposition rate of $0.28 \AA / \mathrm{s}$. This Au capping layer prevents oxidation of the FePd and allows a simpler analysis of the magnetic properties of the system by avoiding substantial effects created by using magnetically polarizable capping materials such as Pd. ${ }^{12,13,14,15}$

Various structural and magnetic characterization techniques were used to investigate these films and the ones relevant to the present study are: X-ray diffraction (XRD), Superconducting Quantum Interference Device magnetometry (SQUID), and magnetic force microscopy (MFM). XRD experiments were performed using a four-circle diffractometer with $\mathrm{Cu} K \alpha$ radiation $(\lambda=1.5418 \AA$ ) in the Bragg-Brentano configuration with $1 / 4$ degree slits. MFM measurements were obtained using a Nanotec microscope in non-contact mode ${ }^{16}$ and with Cr-Co coated pyramidal tips with typical radii of less than $30 \mathrm{~nm}$. The tip was scanned at a constant lift height above the samples (typically $30 \mathrm{~nm}$ ) and the phase shift, which is proportional to the force gradient, was measured to obtain MFM images. SQUID M-H loops were taken with magnetic fields up to $45 \mathrm{kOe}$ applied parallel and perpendicular to the plane of the samples at RT using a Quantum Design MPMS-XL system.

\section{Growth Temperature and Thickness Dependence on FePd Chemical Ordering}

It is well known that the FePd $L 1_{0}$ chemically ordered phase only forms at elevated temperatures during alloy growth in bulk, undergoing an order-disorder transition at $650{ }^{\circ} \mathrm{C}$. ${ }^{17}$ For thin films, Clavero et al. ${ }^{18}$ have shown that the maximum long-range chemical ordering parameter $\left(S_{\text {order }}\right)$ occurs at a deposition temperature of $450{ }^{\circ} \mathrm{C}$ for $22 \mathrm{~nm}$ thick FePd triode sputtered films on $\mathrm{MgO}(001)$, while Kamp et al. ${ }^{19}$ have shown that it occurs at $350{ }^{\circ} \mathrm{C}$ for $30 \mathrm{~nm}$ thick FePd films grown by molecular beam epitaxy (MBE) on $\mathrm{MgO}(001)$. These results evidenced that the deposition technique can significantly affect the maximum ordering temperature in FePd alloys grown on $\mathrm{MgO}(001)$. In our case, dc magnetron sputtering deposition was used, and a series of $40 \mathrm{~nm}$ thick FePd films were grown at deposition temperatures ranging from RT to $600{ }^{\circ} \mathrm{C}$ in order to determine the optimal temperature for chemical ordering.

Gaussian fits of symmetric and asymmetric XRD scans, as well as rocking curves, were used to determine lattice parameters, average grain sizes, mosaic structure, the degree of chemical order $\left(S_{\text {order }}\right)$, and the volume percentage $(\%)$ of $L 1_{0}$ phase in the films, which are shown in Table I. The calculation of the long-range chemical ordering parameter $\left(S_{\text {order }}\right)$, which ranges from $0-1$ for a fully disordered and ordered structure respectively, and the volume percentage (\%) of $L 1_{0}$ phase were calculated using standard procedures described elsewhere. ${ }^{3}$ Symmetric XRD scans for this series of $40 \mathrm{~nm}$ thick FePd films are shown in Fig. 1, where the appearance of the superstructure FePd(001) reflection reveals that chemical ordering occurs between $400-600{ }^{\circ} \mathrm{C}$. These results are similar to those found by Clavero et al. ${ }^{18}$ in that the optimal ordering temperature occurs near $450{ }^{\circ} \mathrm{C}$, where both a high $S_{\text {order }}$ and $\%$ of $L 1_{0}$ phase is formed. We attribute the high degree of chemical order in our films to the pre-treatment of the MgO substrates, which were UHV annealed at high temperature $\left(700{ }^{\circ} \mathrm{C}\right)$ for 30 minutes to 
allow re-crystallization of the surface. Afterwards, the substrates were allowed to cool down to the adequate growth temperature before the actual FePd thin film deposition.

Thus, $450{ }^{\circ} \mathrm{C}$ was considered to be the optimal growth temperature to achieve highly ordered epitaxial FePd thin films using dc magnetron sputter deposition directly on MgO(001) substrates. A thickness dependent FePd film series $(t=5-80 \mathrm{~nm})$ was grown at $450{ }^{\circ} \mathrm{C}$ and we show in Table II that $S_{\text {order }}$ increases linearly with the FePd thickness under identical growth conditions. The lower degree of chemical ordering for thinner films is due to the great lattice mismatch between FePd and MgO (9 \%) that hinders ordering during the early stages of growth, where strain relaxation most likely occurs through microtwins formed by the pileup of partial dislocations across the FePd film thickness. ${ }^{20,21}$

\section{FePd Chemical Ordering and Magnetic Anisotropy Effects on the Magnetic Domain Size/Period}

In order to evaluate the first order uniaxial anisotropy constant $\left(K_{u}\right), \mathrm{M}-\mathrm{H}$ loops were measured at RT with magnetic fields up to $45 \mathrm{kOe}$ applied both perpendicular and parallel to the plane of the sample, i.e. along the easy and hard directions, respectively (see Fig. 2). This allows a calculation of the effective average magnetic anisotropy ( $K_{\text {eff }}$ ) from the area enclosed between the perpendicular and in-plane $\mathrm{M}-\mathrm{H}$ loops, from which $K_{u}$ can be obtained by correcting for the stray field contribution $\left(K_{d}\right)$. $^{22}$

We observe that $K_{u}$ increases with the chemical ordering $\left(S_{\text {order }}\right)$ of the FePd films as indicated in Table II, in agreement with previous observations for strong PMA materials. ${ }^{19}$ The effects of these changes in chemical ordering parameter $\left(S_{\text {order }}\right)$ and the first order uniaxial anisotropy constant $\left(K_{u}\right)$ on the magnetic domain size/period was investigated using an analytical energy model for high anisotropy materials., ${ }^{1,6}$

In contrast with low-anisotropy films $\left(Q_{u}<1\right)$, where a micromagnetic approach is needed to understand the correlation between domain size/period and magnetic anisotropy, analytical models can be used with strong PMA films $\left(Q_{u}>1\right)$ because the domain size is always much larger than the Bloch wall width. The total energy of the magnetic domain pattern (Eq. 1), following the calculation by Kooy and Enz (K-E), ${ }^{6}$ can be written as the sum of three different energy contributions: the domain wall energy, the energy of the magnetization in an external field (Zeeman), and the demagnetizing (stray field) energy.

$$
E_{\text {total }}=D K_{d}\left[\frac{4 \lambda_{c}}{p}-2 h m+m^{2}+\frac{1}{1+\sqrt{\mu}} \frac{8 p}{\pi^{3}} \sum_{n=1}^{\infty} \frac{1}{n^{3}} \operatorname{Sin}^{2}\left[\frac{n \pi(1+m)}{2}\right]\left[1-e^{\frac{-2 n \pi \sqrt{\mu}}{p}}\right]\right]
$$

where $D$ is the FePd film thickness $(\mathrm{m}), K_{d}$ is the stray field energy coefficient $\left(\mathrm{J} / \mathrm{m}^{3}\right), \lambda_{c}$ is the reduced characteristic (dipolar) length, $p$ is the reduced domain period, $h$ is the reduced bias field, $m$ is the reduced magnetization, and $\mu$ is the so-called " $\mu$ method" correction. ${ }^{1}$ Physically the reduced characteristic length $\left(\lambda_{c}\right)$ is a measure of the critical thickness between single- and multi-domain states ${ }^{23}$ and the reduced domain period $(p)$ is the actual size of the magnetic domain period divided by the film thickness $(D)$. In our case, the Zeeman energy term is zero $(h$ $=0$ ) because this model was applied to films in the absence of an applied magnetic field. The system with high perpendicular anisotropy creates smaller up and down domains separated by 
thin walls to minimize the stray field energy, therefore creating more domain walls which raise the total energy. Thus, there exists a delicate energetic balance between these two energy terms for the system to minimize the total energy.

The total energy $\left(E_{\text {total }}\right)$ of the domain pattern (1) can be minimized with respect to the reduced domain period $(p)$ to yield corresponding values of the reduced characteristic length $\left(\lambda_{c}\right)$ as shown in (2).

$$
\lambda_{c}=\frac{2}{1+\sqrt{\mu}} \frac{p^{2}}{\pi^{3}} \sum_{n=1}^{\infty} \frac{1}{n^{3}} \operatorname{Sin}^{2}\left[\frac{n \pi(1+m)}{2}\right]\left[1-\left(1+\frac{2 n \pi \sqrt{\mu}}{p}\right) e^{\frac{-2 n \pi \sqrt{\mu}}{p}}\right]
$$

The magnetic domain structure of these strong PMA films in a demagnetized state $(\mathrm{m}=$ 0 ) was analyzed using magnetic force microscopy (MFM). Although the MFM tip used to image the films does have a stray field, it is not large enough to affect the magnetic domain pattern in these high anisotropy films. MFM images $3 \mu \mathrm{m}$ x $3 \mu \mathrm{m}$ in size were acquired (shown in Fig. 2 insets) and the average magnetic domain size $(D p / 2)$ was calculated using a self-correlation function and the stereological method. ${ }^{24}$ These two different methods were used to ensure correct measurement of the magnetic domain size/period because the domains did not exhibit straight parallel walls, thus making this measurement non-trivial. These two methods showed good agreement $( \pm 3 \%)$ with each other and the average value was used in the analysis. The experimentally measured magnetic domain size/period followed the expected trend indicated by the K-E model.

The experimentally determined reduced domain period $(p)$ was correlated with the experimental reduced characteristic length $\left(\lambda_{c}\right)$, which was calculated according to $\lambda_{c}=$ $4\left(\mathrm{~A} K_{u}\right)^{1 / 2} / 2 D K_{d}$. We find that the resulting experimental data consisting of the measured magnetic domain size/period versus the experimental reduced length $\left(\lambda_{c}\right)$ incorporating $K_{u}$, along with other relevant parameters, agrees well with the proposed analytical energy K-E model considering a delicate balance between domain wall energy and the demagnetizing stray field energy (Fig. 3). Thus, we have found a direct correlation between the magnetic anisotropy, and hence magnetic ordering, and the average magnetic domain size/period in our FePd films which is in good agreement with this analytical energy model.

\section{Conclusions}

We have found that the first order uniaxial anisotropy constant $\left(K_{u}\right)$ in epitaxial FePd thin films grown by dc magnetron sputter deposition at the optimal chemical ordering temperature $\left(450{ }^{\circ} \mathrm{C}\right)$ and with varied thickness $(5-80 \mathrm{~nm})$ is directly correlated with the amount of chemical order $\left(S_{\text {order }}\right)$. In the present case, we propose for the first time a more accurate description of the relevant quantities in chemically ordered binary alloy thin films with strong PMA, i.e. the correlation between microstructure (amount of chemical order) and magnetic anisotropy, and hence domain pattern characteristics. The K-E model, a balance between domain wall energy and demagnetizing stray field energy, showed good agreement with the experimentally measured quantities allowing a correlation between magnetic anisotropy and domain size/period. These results are relevant for the development and design of future ultrahigh density perpendicular media. 


\section{Acknowledgment}

The authors acknowledge K. Seo, J. Lu, and S. A. Wolf for technical collaboration. Funding from the Virginia Space Grant Consortium, National Science Foundation (DMR0355171), Research Corporation (Cottrell Scholar Award), and the American Chemical Society (PRF-41319-AC10) is acknowledged.

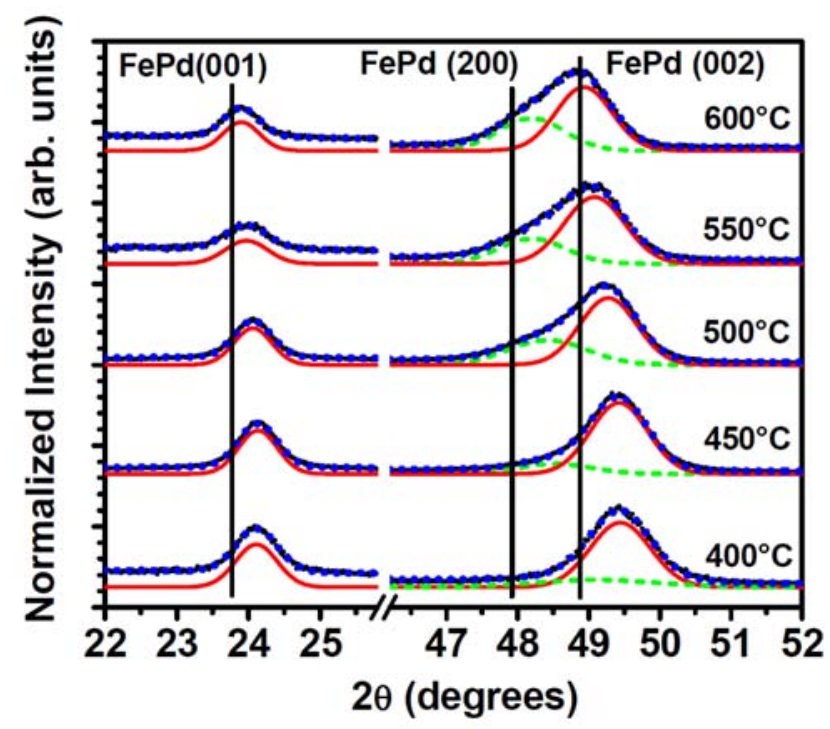

FIG. 1. (color online) Symmetric XRD scans showing $L 1_{0}$ chemical ordering for the FePd film series with fixed thickness $(40 \mathrm{~nm})$ and deposited at temperatures ranging from $400-600{ }^{\circ} \mathrm{C}$ (black data). Gaussian fits for the superlattice FePd(001) and fundamental FePd(002) reflections indicate the presence of chemical ordering (red solid lines), whereas FePd(200) reflections indicate the face-centered-cubic ( $f c c$ ) disordered phase (green dashed lines). Cumulative fits (blue dotted lines) are shown whereas background fits have been removed for clarity. Vertical lines denote bulk lattice parameters. 
TABLE I

MicrostruCtURAL PARAMETERS EXTRACTED FROM XRD ANALYSIS FOR THE FEPD FILM SERIES GROWN AT DiFFERENT TEMPERATURES

\begin{tabular}{cccccccc}
\hline \hline $\begin{array}{c}\text { FePd Growth } \\
\text { Temperature }\left({ }^{\circ} \mathrm{C}\right)\end{array}$ & $\begin{array}{c}c(\AA) \\
( \pm 0.001 \AA)\end{array}$ & $\begin{array}{c}a(\AA) \\
( \pm 0.001 \AA)\end{array}$ & $\begin{array}{c}c / a \\
( \pm 0.0005)\end{array}$ & $\begin{array}{c}\text { Average } \\
\text { Grain Size }(\AA) \\
( \pm 1.0 \AA)\end{array}$ & $\begin{array}{c}\text { Mosaic Structure } \\
\text { (degrees) } \\
( \pm 0.01 \text { degrees })\end{array}$ & $\begin{array}{c}S_{\text {order }} \\
\% \text { of } L 1_{0} \text { phase } \\
\pm 1 \%)\end{array}$ \\
\hline 400 & 3.688 & 3.852 & 0.9574 & 119 & 2.60 & $0.80 \pm 0.03$ & $75 \%$ \\
450 & 3.685 & 3.851 & 0.9569 & 127 & 2.63 & $0.75 \pm 0.04$ & $0.77 \pm 0.04$ \\
500 & 3.696 & 3.855 & 0.9588 & 128 & 2.27 & 2.58 & $0.58 \pm 0.04$ \\
550 & 3.709 & 3.847 & 0.9641 & 117 & 138 & 2.13 & $0.62 \pm 0.04$
\end{tabular}

Perpendicular (c) and in-plane (a) lattice parameters, c/a ratio, average grain size, and mosaic structure for the $L 1_{0}$-ordered phase extracted from XRD analysis. $S_{\text {order }}$ and the $\%$ of $L 1_{0}$ phase is also calculated in the FePd film series grown at different deposition temperatures with fixed thickness (40 nm).

TABLE II

MEASURED VALUeS OF $S_{\text {ORDER }}$ AND $K_{U}$ FOR THE FePd THICKNESS SERIES

\begin{tabular}{ccc}
\hline \hline$t(\mathrm{~nm})$ & $S_{\text {order }}$ & $K_{u}\left(\mathrm{~J} / \mathrm{m}^{3}\right)( \pm 2 \%)$ \\
\hline 5 & -- & $9.56 \times 10^{5}$ \\
10 & $0.58 \pm 0.19$ & $9.60 \times 10^{5}$ \\
20 & $0.64 \pm 0.06$ & $1.04 \times 10^{6}$ \\
30 & $0.65 \pm 0.04$ & $1.07 \times 10^{6}$ \\
40 & $0.75 \pm 0.04$ & $1.10 \times 10^{6}$ \\
80 & $0.82 \pm 0.02$ & $1.24 \times 10^{6}$ \\
\hline
\end{tabular}

Chemical ordering parameters $\left(S_{\text {order }}\right)$ and first order uniaxial anisotropy constants $\left(K_{u}\right)$ for the FePd film series grown $450{ }^{\circ} \mathrm{C}$ with varied thickness $(t$ $=5-80 \mathrm{~nm}$ ). Linear trends can be evidenced between $t, S_{\text {order }}$, and $K_{u}$. 


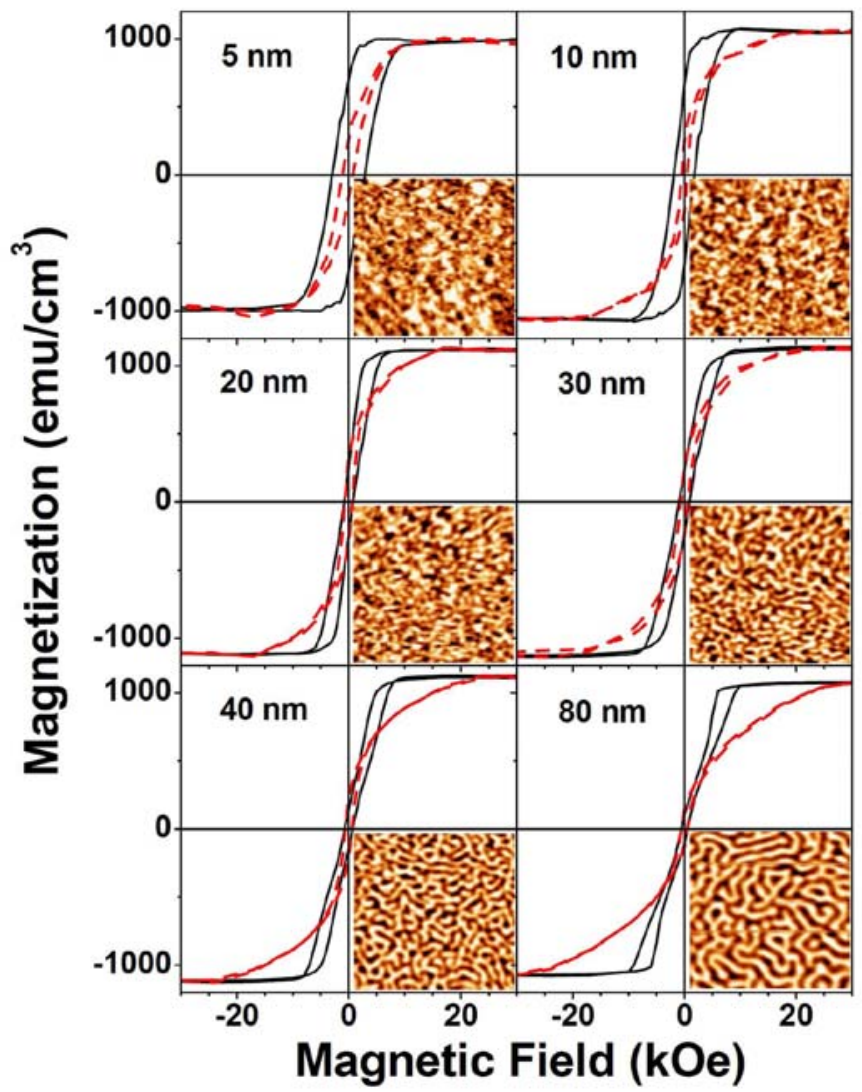

FIG. 2. (color online) In-plane (red dashed lines) and perpendicular (black solid lines) M-H loops for the thickness dependent $(5-80 \mathrm{~nm})$ FePd series deposited at the optimal chemical ordering temperature $\left(450^{\circ} \mathrm{C}\right.$ ). Corresponding MFM images ( $3 \mu \mathrm{m} \times 3 \mu \mathrm{m}$ in size) are shown in the insets. 


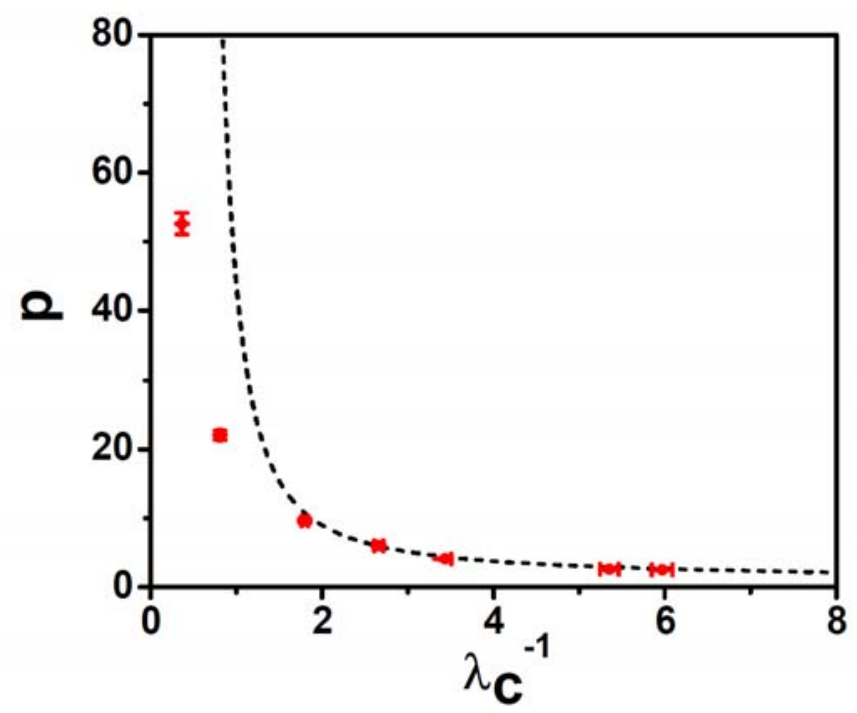

FIG. 3. The reduced domain period $(p)$, measured from MFM images, is correlated with the reduced characteristic length $\left(\lambda_{c}\right)$, calculated from $K_{u}$ and other relevant parameters, and shows good agreement (red data) with the K-E analytical energy model (black dashed line). 


\section{References}

${ }^{1}$ A. Hubert and R. Schafer, Magnetic Domains The Analysis of Magnetic Microstructures (Springer, Berlin, 2000), pp. 107-354.

${ }^{2}$ D. Weller and A. Moser, IEEE Trans. Magn. 35, 4423 (1999).

${ }^{3}$ A. Cebollada, R. F. C. Farrow, and M. F. Toney, Magnetic Nanostructures, edited by H. S. Nalwa (American Scientific Publishers, 2002).

${ }^{4}$ R. Skomski, A. Kashyap, and J. Zhou, Scripta Materialia 53, 389 (2005).

${ }^{5}$ N. Honda and K. Ouchi, J. Magn. Magn. Mater. 235, 289 (2001).

${ }^{6}$ C. Kooy and U. Enz, Philips Res. Repts 15, 7 (1960).

${ }^{7}$ V. Gehanno, A. Marty, B. Gilles, and Y. Samson, Phys. Rev. B 55, 12552 (1997).

${ }^{8}$ V. Gehanno, Y. Samson, A. Marty, B. Gilles, and A. Chamberod, J. Magn. Magn. Mater. 172, 26 (1997).

${ }^{9}$ A. Asenjo, J. M. Garcia, D. Garcia, A. Hernando, M. Vazquez, P. A. Caro, D. Ravelosona, A. Cebollada, and F. Briones, J. Magn. Magn. Mater. 196-197, 23 (1999).

${ }^{10}$ Y. Samson, A. Marty, R. Hoffmann, V. Gehanno, and B. Gilles, J. Appl. Phys. 85, 4604 (1998).

${ }^{11}$ E. B. Svedberg, P. Sandstrom, J.-E. Sundgren, J. E. Greene, and L. D. Madsen, Surf. Sci. 429, 206 (1999).

${ }^{12}$ C. Clavero, J. R. Skuza, Y. Choi, D. Haskel, J. M. Garcia-Martin, A. Cebollada, and R. A. Lukaszew, Appl. Phys. Lett. 92, 162502 (2008).

${ }^{13}$ Y. Huttel, E. Navarro, N. D. Telling, G. van der Laan, F. J. Palomares, C. Quintana, E. Roman, G. Armelles, and A. Cebollada, Phys. Rev. B 78, 104403 (2008).

${ }^{14}$ E. Navarro, Y. Huttel, C. Clavero, A. Cebollada, and G. Armelles, Phys. Rev. B 69, 224419 (2004). 
${ }^{15}$ E. Navarro, Y. Huttel, C. Clavero, G. Armelles, and A. Cebollada, Appl. Phys. Lett. 84, 2139 (2004).

${ }^{16}$ I. Horcas, R. Fernandez, J. M. Gomez-Rodriguez, J. Colchero, J. Gomez-Herrero, and A. M. Baro, Rev. Sci. Instr. 78, 013705 (2007).

17 T. B. Massalski, H. Okamoto, P. R. Subramanian, and L. Kacprzak (eds.), Binary Alloy Phase Diagrams, second ed. (ASM International, Materials Park, OH, 1990), p. 1751.

${ }^{18}$ C. Clavero, J. M. Garcia-Martin, J. L. Costa Kramer, G. Armellas, A. Cebollada, Y. Huttel, R. A. Lukaszew, and A. J. Kellock, Phys. Rev. B 73, 174405 (2006).

${ }^{19}$ P. Kamp, A. Marty, B. Gilles, R. Hoffman, S. Marchesini, M. Belakhovsky, C. Boeglin, H. A. Durr, S. S. Dhesi, G. van der Laan, and A. Rogalev, Phys. Rev. B 59, 1105 (1999).

${ }^{20}$ D. Halley, Y. Samson, A. Marty, P. Bayle-Guillemaud, C. Beigne, B. Gilles, and J. E. Mazille, Phys. Rev. B 65, 205408 (2002).

${ }^{21}$ D. Halley, A. Marty, P. Bayle-Guillemaud, B. Gilles, J. P. Attane, and Y. Samson, Phys. Rev. B 70, 174438 (2004).

${ }^{22}$ O. Ersen, V. Parasote, V. Pierron-Bohnes, M. C. Cadeville, and C. Ulhaq-Bouillet, J. Appl. Phys. 93, 2987 (2003).

${ }^{23}$ C. Kittel, Phys. Rev. 70, 965 (1946).

${ }^{24}$ R. Bodenberger and A. Hubert, Phys. Status Solidi A 44, K7-11 (1977). 